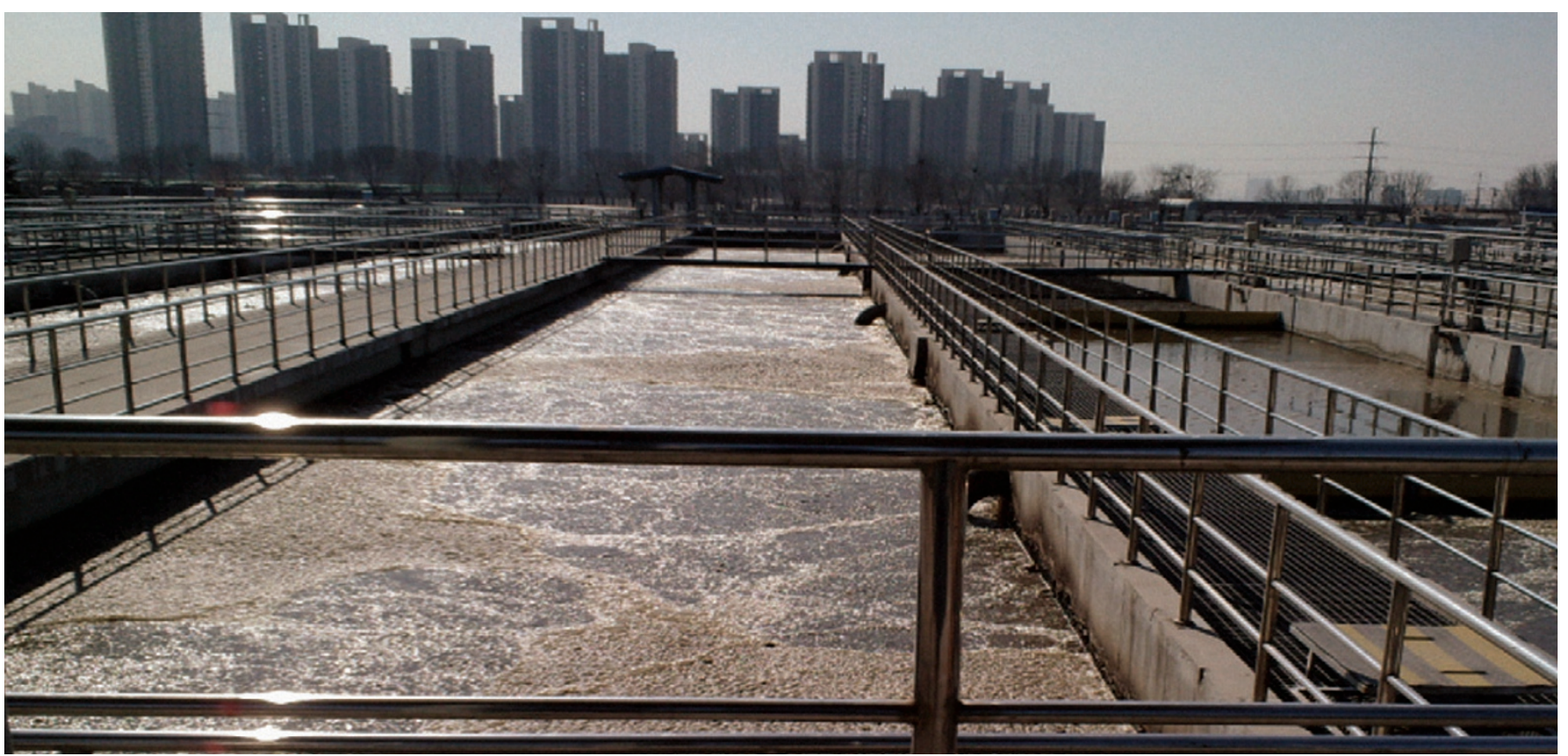

Enzymes that trigger antibiotic resistance can survive water treatment plants on China's Haihe River.

\title{
DIAGNOSTICS
}

\section{Detection drives defence}

\section{Devices that quickly identify bacterial infections would benefit health and slow the spread of resistance.}

\section{BY REBECCA KANTHOR}

$\mathrm{I}$ n northern China, five large rivers and more than 300 smaller ones flow into the Haihe River, known as the 'mother river' of Tianjin, one of the country's largest cities. But something unpleasant has entered the river too: potentially deadly genes. In 2010, researchers from Nankai University in Tianjin reported finding genes in the Haihe River that can make bacteria resistant to antibiotics.

But it gets worse. A group of scientists from three universities recently found that New Delhi metallo-beta-lactamase (NDM-1) an enzyme that can make bacteria resistant to a wide range of antibiotics - has survived the sanitizing processes of water treatment plants on the river ${ }^{1}$. The Haihe River exemplifies China's problems with antibiotic resistance and the need for improved diagnostics to track these life-threatening bacteria. Battling problems like this one, and the related health-care challenges, demands better tools for measuring antibiotic resistance around the world.

It may be no surprise to find antibioticresistant genes in China's waters, because China is the largest producer and consumer of antibiotics in the world. Wang Liping, a microbiologist at Shanghai's Jiao Tong University, describes the scene in most local hospitals: "You will see many people receiving an intravenous injection. If you have just a common cold but you have a higher temperature, you will be on antibiotics for sure."

Part of the problem is that antibiotics contribute to the financial stability of China's hospitals. Xiao Yonghong, who studies infectious diseases at Zhejiang University and directs China's Antibiotic Resistance Monitoring Network, says that antibiotics account for $20 \%$ of hospital drug sales, and that pharmaceutical companies offer incentives for physicians to prescribe them.

Better tests to help doctors diagnose infections could make a big difference to the way patients are treated in China, says Xiao. Faced with a patient with flu-like symptoms, many clinicians now prescribe broad-spectrum antibiotics as they wait several days for lab results to determine whether the infection is bacterial or viral. Advanced diagnostics, Xiao says, would help "to decrease the misuse of antibiotics". Diagnostic tools that speed up the identification of infections "would help doctors to make the right decisions about antibiotics".

Without these tools, resistance to antibiotics is increasing, and many so-called superbugs are on the rise, including methicillin-resistant
Staphylococcus aureus (MRSA) and other difficult-to-treat microorganisms, such as extended-spectrum beta-lactamase-producing Escherichia coli and multidrug-resistant Acinetobacter. "During the past 10 years, antibiotic resistance rates have increased sharply in China," says Xiao. "This is very common in hospital settings." Resistance rates of E. coli and MRSA have more than doubled in China since 2000 , he says, rising from $10 \%$ to $60 \%$, and from $20 \%$ to $50 \%$, respectively.

But China's problems with antibiotics are spreading beyond its borders. "With people travelling and tourism, resistance in one country could spread to other countries," Xiao says. "I don't think it is a local problem - it is a global problem."

\section{A SIGN OF SEPSIS}

As well as helping clinicians reduce the use of antibiotics, advanced diagnostics can also show when they are needed. In some cases, early diagnosis can make the difference between life and death. For example, sepsis is a whole-body infection, usually caused by bacteria, that is estimated to infect 4 million people a year, although
D NATURE.COM

Recent work

on rapid

diagnosis:

go.nature.com/rJvTqg 
global prevalence rates are uncertain. Sources indicate that at least $30 \%$ of people who get sepsis will die, often because treatment comes too late. According to the Sepsis Alliance in San Diego, California: "Sepsis should be treated as a medical emergency" requiring "rapid administration of antibiotics and fluids." The Alliance's website adds: "A 2006 study showed that the risk of death from sepsis increases by $7.6 \%$ with every hour that passes before treatment begins."

In China, sepsis in young children is a particular problem. Neonatal sepsis affects up to $15 \%$ of children up to three months of age in developing countries, and Chinese researchers have found ${ }^{2}$ that "early diagnosis and treatment of neonatal sepsis is especially vital in China."

One way of diagnosing sepsis is to measure the level of procalcitonin (PCT) in the blood. In healthy people, high levels of calcium in the blood trigger the production of PCT, which is a precursor to calcitonin, a hormone that causes the body to reduce the calcium level. A bacterial infection, however, causes cells throughout the body to ramp up PCT production as part of the immune response. For a patient with sepsis, the level of PCT in the blood rises within a couple of hours of being infected and can be used to indicate the severity of infection. The difficulty is finding an easy way to measure it.

One rapid solution is the BRAHMS PCT-Q assay produced by Thermo Fisher Scientific in Waltham, Massachusetts. In this assay, a gold tracer binds to PCT so it can be quantified. One of the assay's benefits is its speed: in less than an hour, clinicians can distinguish between bacterial and viral infections, which do not raise PCT levels. During treatment, clinicians can also use the assay to determine when it is safe to end the course of antibiotics.

Improved diagnostics can also help clinicians assess the danger from previously ignored infections. For example, many countries track Clostridium difficile, a bacteria that infects the colon, but many Asian countries disregard it. A recent review of infections in Asia, however, concluded $^{3}$ that $C$. difficile infection "could be widespread in those regions where surveillance is currently lacking. Asia may be facing a 'perfect storm' as heavy usage of antibiotics combines with an ageing, increasingly hospitalized population."

One approach for detecting $C$. difficile is an assay developed by Quidel, based in San Diego, California. "The gold-standard culture method takes four days and a lot of work," says Timo-

“Instead of
identifying just
one infection,
tomorrow's
tools could
provide broader
diagnostic
possibilities."
thy Stenzel, Quidel's chief scientific officer. "What patient wants to wait four days to determine the cause of their potentially life-threatening diarrhoea?" Instead, he says, the Quidel test provides an answer in just two hours. Slowing down a bacterial epidemic takes that kind of speed, but there are many more bacterial infections than sepsis and C. difficile.

\section{A BROADER BASIS}

Instead of identifying just one infection, tomorrow's tools could provide broader diagnostic possibilities. Ralph Weissleder, who studies inflammatory diseases and is director of the Center for Systems Biology at Massachusetts General Hospital, uses genetic markers - parts of the bacterial genome - to detect bacterial infections. These markers can identify virtually any bacteria. This is particularly difficult when bacterial numbers are low. "As with most of the bacterial detection devices, the challenge is always detecting a few 'bad' bacteria in a sea of 'good' bacteria," Weissleder says.

So far, the team has tested the technology by identifying tuberculosis ${ }^{4}$, searching for gene sequences unique to Mycobacterium tuberculosis. A patient's blood sample, or other body fluid, is placed into the device, which uses the polymerase chain reaction to make more copies of bacterial DNA, making it easier to identify (see 'Infection-detecting device'). Weissleder

\section{INFECTION-DETECTING DEVICE}

In this microfluidic chip, bacterial DNA attached to beads is labelled with magnetic nanoprobes, mixed with a buffer in channels, and a nuclear magnetic resonance (NMR) microcoil identifies the infection.

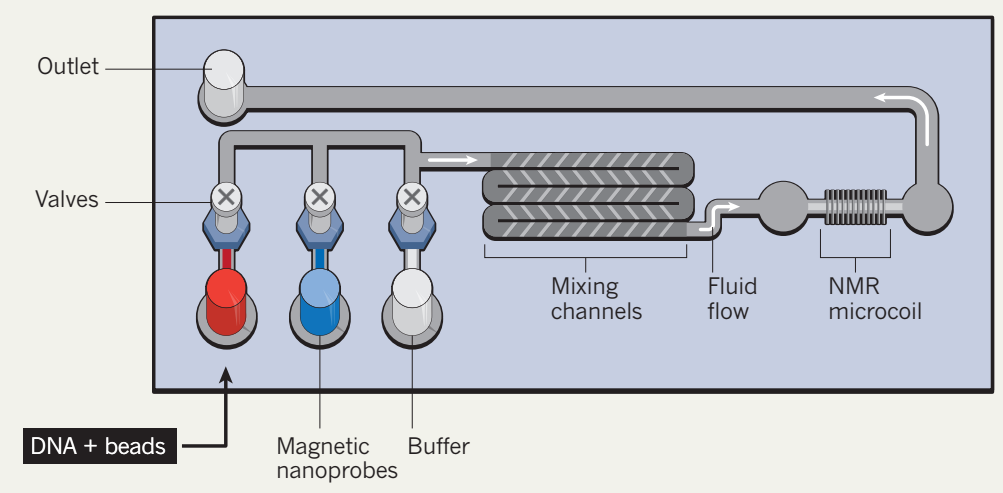

hopes the kit will be accessible to doctors all over the world, including in underdeveloped regions. The kit is little thicker than a microscope slide and the sensors are inexpensive to make. "In two hours you know exactly what kind of bacteria are in there and what the resistance profiles are." This technology can also identify many other bacteria, provided they have unique gene sequences. T2 Biosystems, a company co-founded by Weissleder, is developing the technology into a medical device, which is in phase III clinical trials.

Success in one medical arena can come from failures in another. For 25 years, Nicholas Topley at Cardiff University, UK, has worked with kidney-failure patients on a dialysis process using peritoneal fluid, which provides lubrication between the organs and body wall in the abdomen. Working with Matthias Eberl, an immunologist in his lab, Topley realized that peritoneal fluid contains evidence of infections that afflict dialysis patients. This means the pair could "look at whether the biomarkers we measure actually predict the type of pathogen," Eberl says. By seeing which biological markers in the peritoneal fluid have changed, Eberl and Topley hope to find a 'fingerprint' that identifies each kind of pathogen. They have already found that analysing the peritoneal fluid can distinguish some types of bacteria ${ }^{5}$.

As well as helping patients with kidney failure, this technology could be used for bacterial infections in general. The team is collaborating with Mologic, a biotech company in Bedford, UK, to develop an at-home dipstick test for kidney-failure patients, which would appear and function similar to a pregnancy test. "It would gain precious time as people could basically diagnose themselves and see if they have an infection," Eberl says. "They could then go directly to the hospital and get the most appropriate treatment." This could improve the effectiveness of the treatment and reduce the chance of the bacteria developing resistance.

Improving the accuracy and speed of bacterial diagnostics around the world could have an enormous impact. The potential to save lives with faster and more targeted diagnoses, decrease unnecessary and often incorrect prescriptions, and even help identify early on where bacterial resistance could occur, will have a drastic effect on the way patients are treated. For these tools to do their jobs, though, they must be accessible to the doctors and patients who need them. As Liping says of using advanced bacterial diagnostics: "It can be a life-saving situation in many cases."

\section{Rebecca Kanthor is a freelance journalist} based in Shanghai, China.

1. Luo, Y etal. Environ Sci. Technol Lett 1, 26-30 (2014)

2. Li, Z et al. Int J Clin Exp Med 6. 693-699 (2013).

3. Collins, D. A., Hawkey, P. M. \& Riley, T. V. Antimicrob. Resist. Infect. Control 2, 21 (2013).

4. Liong, M. et al. Nature Commun. 4, 1752 (2013).

5. Lin, C.-Y. et al. J. Am. Soc. Nephrol. 24, 2002-2009 (2013). 